\title{
Article \\ Quality of Life as a Mediator between Cancer Stage and Long-Term Mortality in Nasopharyngeal Cancer Patients Treated with Intensity-Modulated Radiotherapy
}

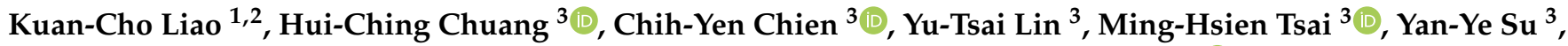 \\ Chao-Hui Yang ${ }^{3}$, Chi-Chih Lai ${ }^{3}$, Tai-Lin Huang ${ }^{4}$, Shau-Hsuan Li ${ }^{4}$, Tsair-Fwu Lee ${ }^{5,6} \mathbb{D}$, Wei-Ting Lin ${ }^{7}$, \\ Chien-Hung Lee $2,8,9,10, *,+\mathbb{D}$ and Fu-Min Fang $1,11, *,+$
}

check for updates

Citation: Liao, K.-C.; Chuang, H.-C.; Chien, C.-Y.; Lin, Y.-T.; Tsai, M.-H.; Su, Y.-Y.; Yang, C.-H.; Lai, C.-C.; Huang, T.-L.; Li, S.-H.; et al. Quality of Life as a Mediator between Cancer Stage and Long-Term Mortality in

Nasopharyngeal Cancer Patients

Treated with Intensity-Modulated Radiotherapy. Cancers 2021, 13, 5063. https://doi.org/10.3390/

cancers13205063

Academic Editors

Alessandra Rinaldo and David Wong

Received: 3 August 2021

Accepted: 8 October 2021

Published: 10 October 2021

Publisher's Note: MDPI stays neutral with regard to jurisdictional claims in published maps and institutional affiliations.

Copyright: (C) 2021 by the authors Licensee MDPI, Basel, Switzerland. This article is an open access article distributed under the terms and conditions of the Creative Commons Attribution (CC BY) license (https:/ / creativecommons.org/licenses/by/ $4.0 /)$.
1 Department of Radiation Oncology, Kaohsiung Chang-Gung Memorial Hospital and Chang Gung University College of Medicine, Kaohsiung 833401, Taiwan; piko@cgmh.org.tw

2 Department of Public Health, College of Health Sciences, Kaohsiung Medical University, Kaohsiung 807378, Taiwan

3 Department of Otolaryngologist, Kaohsiung Chang-Gung Memorial Hospital and Chang Gung University College of Medicine, Kaohsiung 833401, Taiwan; entjulia@cgmh.org.tw (H.-C.C.); cychien3965@adm.cgmh.org.tw (C.-Y.C.); xeye@cgmh.org.tw (Y.-T.L.); b9302094@cgmh.org.tw (M.-H.T.); yanyesu@cgmh.org.tw (Y.-Y.S.); chingmn@cgmh.org.tw (C.-H.Y.); gordon93@cgmh.org.tw (C.-C.L.)

4 Department of Hematology and Oncology, Kaohsiung Chang-Gung Memorial Hospital and Chang Gung University College of Medicine, Kaohsiung 833401, Taiwan; victor99@cgmh.org.tw (T.-L.H.); lee0624@cgmh.org.tw (S.-H.L.)

5 Medical Physics and Informatics Laboratory of Electronics Engineering, National Kaohsiung University of Science and Technology, Kaohsiung 80778, Taiwan; tflee@nkust.edu.tw

6 Department of Medical Imaging and Radiological Sciences, Kaohsiung Medical University, Kaohsiung 807378, Taiwan

7 Department of Social, Behavioral and Population Sciences, School of Public Health and Tropical Medicine, Tulane University, New Orleans, LA 70112, USA; lweiting@tulane.edu

8 Research Center for Environmental Medicine, Kaohsiung Medical University, Kaohsiung 807378, Taiwan

9 Department of Medical Research, Kaohsiung Medical University Hospital, Kaohsiung Medical University, Kaohsiung 807378, Taiwan

10 Office of Institutional Research \& Planning, Secretariat, Kaohsiung Medical University, Kaohsiung 807378, Taiwan

11 Department of Medicine, Chang Gung University College of Medicine, Taoyuan 333323, Taiwan

* Correspondence: cnhung@kmu.edu.tw (C.-H.L.); fang2569@cgmh.org.tw (F.-M.F.); Tel.: +886-7-312-1101 (ext. 2314) (C.-H.L.); +886-7-731-7123 (ext. 7000) (F.-M.F.); Fax: +886-7-311-0811 (C.-H.L.); +886-7-398-6179 (F.-M.F.)

+ These authors contributed equally to this work.

Simple Summary: Even after the implementation of intensity-modulated radiotherapy (IMRT), nasopharyngeal cancer (NPC) survivors may continue to exhibit several physical symptoms that negatively affect long-term quality of life $(\mathrm{QoL})$. An NPC patient cohort study $(n=682)$ was conducted to examine the potential mediating effect of QoL (evaluated at multiple treatment-related time points) on the cancer stage-mortality association. Patients with advanced NPC exhibited low global health QoL and high QoL-HN35 symptom pre-IMRT, 3 months post-IMRT, and 2 years post-IMRT. Global health QoL and QoL-HN35 symptom scores 2 years after IMRT explained $49.4 \%$ and $39.4 \%$ of the excessive effect of advanced NPC on mortality risk. Our findings indicate that global health QoL and QoL-HN35 symptom 2 years after IMRT are key mediators of the relationship between advanced NPC and high mortality. These findings emphasize the significance of QoL-HN35 symptom and global health QoL-associated medical support and care for patients with NPC who received IMRT.

Abstract: Background: Quality of life (QoL) attained before, during, or after treatments is recognized as a vital factor associated with therapeutic benefits in cancer patients. This nasopharyngeal cancer (NPC) patient longitudinal study assessed the relationship among QoL, cancer stage, and longterm mortality in patients with nasopharyngeal carcinoma (NPC) treated with intensity-modulated radiotherapy (IMRT). Patients and Methods: The European Organization for Research and Treatment 
of Cancer (EORTC) core QoL questionnaire (QLQ-C30) and the head and neck cancer-specific QoL questionnaire module (QLQ-HN35) were employed to evaluate four-dimensional QoL outcomes at five time points: pre- $(n=682)$, during (around $40 \mathrm{~Gy})(n=675), 3$ months $(n=640), 1$ year $(n=578)$ and 2 years post-IMRT $(n=505)$, respectively, for 682 newly diagnosed NPC patients treated between 2003 and 2017 at a single institute. The median followed-up time was 7.5 years, ranging from 0.3 to 16.1 years. Generalized estimating equations, multivariable proportional hazards models, and Baron and Kenny's method were used to assess the investigated effects. Results: Advanced AJCC stage (III-IV) patients revealed a 2.26-fold (95\% CI-1.56 to 3.27) higher covariate-adjusted mortality risk than early-stage (I-II) patients. Compared with during IMRT, advanced-stage patients had a significantly low global health QoL and a significantly high QoL-HN35 symptom by a large magnitude at pre-, 3 months, and 2 years post-IMRT. QoL scales at pre-IMRT, 1 year, and 2 years post-IMRT were significantly associated with mortality. The effect changes of mortality risk explained by global health QoL, QoL-C30, and QoL-HN35 symptom were 5.8-9.8\% at pre-IMRT but at 2 years post-IMRT were $39.4-49.4 \%$ by global health QoL and QoL-HN35 symptoms. Conclusions: We concluded advanced cancer stage correlates with a long-term high mortality in NPC patients treated with IMRT and the association is partially intermediated by QoL at pre-IMRT and 2 years post-IMRT. Therefore, QoL-HN35 symptom and global health QoL-dependent medical support and care should be focused and tailored at 2 years post-IMRT.

Keywords: quality of life; mediator; nasopharyngeal carcinoma; intensity modulated radiotherapy; mortality; Baron and Kenny's method

\section{Introduction}

In recent years, the medical application of intensity-modulated radiotherapy (IMRT) and optimization of chemotherapy strategies have prominently prolonged survival and lessened toxicities to normal tissues for patients with nasopharyngeal cancer (NPC) [1] However, the 5-year survival rate for advanced-stage NPC (stages III and IV) is still at an ameliorable level, both in Asian areas (e.g., 23-79\% in Japan and $60-79 \%$ in Taiwan) and European areas (e.g., 31-55\% in Finland) [2-4]. Because the current anatomy-based staging system is insufficient for predicting therapeutic benefits or clinical prognosis [1], investigations that consider other clinical and non-clinical factors to help survival prediction are warranted.

Quality of life (QoL) attained before, during, or after treatments is recognized as a vital factor associated with therapeutic benefits in cancer patients [5-11]. Clinical investigations have demonstrated that even with the implementation of intensity-modulated radiotherapy (IMRT), NPC survivors still suffered several physical symptoms that influence long-term QoL $[12,13]$. Patients with advanced-stage NPC were identified as having poorer QoL than early-stage patients [13-15]. Alternatively, QoL has been considered a potential survival predictor for cancer patients. In longitudinal studies of NPC patients with QoL measured pretreatment and 1 year after treatment, an increase in physical functioning and a decrease in fatigue and appetite loss were identified to predict a higher overall survival $[9,10]$.

Studies have reported that approximately $69.0-88.2 \%$ of patients with NPC have already suffered from stage III or IV tumors at diagnosis [11,16-18]. Because advancedstage NPC was associated with a lower QoL score and an inferior level of QoL was linked to a higher mortality, this raised the issues of what time periods of QoL in the treatment and follow-up course and what types of QoL can mediate the effect of advanced cancer stage on long-term mortality for NPC patients treated with IMRT. Hence, the purpose of this longitudinal study of a patient cohort was to investigate the relationship among cancer stage, QoL, and long-term mortality in NPC patients treated with IMRT, and to evaluate the potential mediating effect of QoL evaluated at multiple treatment-related time points on the association of cancer stage with NPC patient mortality. 


\section{Patients and Methods}

\subsection{Patient Data}

Pathology-confirmed NPC patients, newly diagnosed at the Kaohsiung Chang-Gung Memorial Hospital with previously untreated, non-recurrence, and non-distant metastatic status between April 2003 and December 2017 were enrolled. NPC patients with previous or synchronous malignancies $(n=8)$, metastasis at diagnosis $(n=15)$, incapable of completing the prescribed treatment course of IMRT $(n=19)$, and unable to complete the QoL questionnaires at the time point of pre-IMRT $(n=28)$ were excluded. A total of 682 consecutively recruited NPC patients aged 20-80 years participated in this longitudinal study. Among the participants, 65 (9.5\%) and $167(24.5 \%)$ had AJCC stage I and II, and $244(35.8 \%)$ and $206(30.2 \%)$ had AJCC stage III and IV, determined by the 8th American Joint Committee on Cancer (AJCC) staging system [19].

\subsection{Treatment}

The technical details of IMRT in the institute during the study period have been presented in previous reports [9,20]. Before 2011, seven-fixed-beam IMRT with two-phase sequential planning was applied in 436 (63.9\%) patients. The median prescribed dose to the gross tumor and nodal area was 70.2 Gy (range 62.4-78.6 Gy) and to the elective risky area was 45.0-59.4 Gy, with a daily fraction of 1.8 or 2.0 Gy and five fractions per week. After 2011, rotational-arc IMRT with simultaneously integrated boost planning was applied in 246 (36.1\%) patients, with three dose levels of 69.96, 59.4, and 52.8 Gy in 33 fractions prescribed to the high, intermediate, and low clinical target area, respectively. Cisplatin-based chemotherapy was given weekly, intravenously, during the course of IMRT as a radiation sensitizer for those with clinical stages II-IV. Neoadjuvant or adjuvant chemotherapy with the combination regimens of cisplatin and 5-fluorouracil administered every 3-4 weeks was given for 1-4 cycles to those patients with clinical stages III-IV or receiving inadequate doses of cisplatin during the course of IMRT.

\subsection{Follow-Up}

In our treatment network, NPC patients were scheduled to visit the tumor clinics every 3 months after IMRT for primary assessment of treatment effect in the first 2 years, and for regular image and physical examinations every 4-6 months in the third to fifth years and bi-annually in the fifth to tenth years, respectively. After 10 years, patients were followed up year after year for their health condition in the clinics or through telephone interviews. Health insurance data, medical charts, and death certificates were used to determine the vital status and the date of death where relevant for each patient. The follow-up of the patient cohort started on 1 April 2003 and finished on 31 December 2019 (censoring date). Time at risk was defined as the time from the date of tumor stage diagnosis to the date of death or censoring date. The median followed-up time was 7.5 years, ranging from 0.3 to 16.1 years.

\subsection{QoL Instruments}

The core QoL questionnaire (QLQ-C30) and the head and neck cancer-specific QoL questionnaire module (QLQ-HN35) developed by the European Organization for Research and Treatment of Cancer (EORTC, Brussels, Belgium), Taiwan Chinese version 3, were used to collect QoL data from each patient [21]. EORTC QLQ-C30 comprises a scale for global health status, five scales for multidimensional functioning (physical, cognitive, role, emotional, and social functioning), and nine scales/items for disease symptoms (fatigue, nausea, and vomiting, pain, dyspnea, insomnia, diarrhea, constipation, appetite loss, and financial difficulties) [22]. EORTC QLQ-HN35 includes seven symptom scales (problems of pain, swallowing, speech, senses, social contact, social eating, and sexuality), six symptom items (trouble with opening the mouth, teeth, sticky saliva, dry mouth, coughing, and feeling ill), and five dichotomous items (use of painkillers, nutritional supplements, and feeding tube, and weight loss or weight gain). Except for five QLQ-HN35 
dichotomous items and one QLQ-C30 seven-point global health scale, all scales/items were structured in a four-point response. The responses were linearly converted to a score between 0 and 100 [22]. A high score implies a high level of global health status, functioning, and symptoms or problems. In EORTC QLQ-C30, the average scores of the global health scale, functional scales, and symptom scales/items were used to evaluate the "global health QoL", "functioning QoL" and general cancer symptom-related QoL ("QoL-C30 symptom"), respectively. In EORTC QLQ-HN35, the average score of symptom scales/items was used to assess the QoL of head and neck cancer-related symptoms ("QoL-HN35 symptom"). The pre-IMRT was defined as the period during which NPC patients agreed to participate in this study and before they started the first fraction of IMRT treatment. This period was approximately 1 to 2 weeks. Because significant changes in QoL of NPC patients appear within 2 years after IMRT [23], we evaluated the four scales of QoL levels for each patient at five IMRT-associated time points: pre-IMRT $(n=682)$; during IMRT (around $40 \mathrm{~Gy}$ ) $(n=675), 3$ months post-IMRT ( $n=640)$; 1 -year post-IMRT $(n=578)$; and 2 years post-IMRT $(n=505)$.

\subsection{Covariates}

Demographic and clinical variables were obtained from each NPC patient at the initial clinical treatment. Ethnicity was grouped as Minnan and others (including, Hakka, Mainlander, and aborigines). Educational level was classified as $\leq 12$ and $>12$ years. Body mass index (BMI) was categorized as underweight $(\mathrm{BMI}<18.5)$, normal weight $(\mathrm{BMI}=18.5-23.9)$, and overweight (BMI $\geq 24.0$ ) according to the criteria of the Taiwan Health Promotion Administration [24]. Comorbidity was classified as 0 and $\geq 1$ comorbid condition, according to Deyo's Charlson Comorbidity Index (CCI) scoring [25]. The treatment in combination with chemotherapy was recorded as Yes or No.

\subsection{Statistical Analysis}

Proportions were employed to describe the distributions of demographic and clinical factors, and Cox proportional hazards models were used to assess the relationship between these factors and mortality. Overall survival curves were estimated using the Kaplan-Meier method, and the log-rank test was used to evaluate the difference in mortality rates across four clinical stages. We applied mortality density to express the incidence of mortality associated with the clinical stage. Age, gender, ethnicity, educational level, BMI, CCI, chemotherapy, and IMRT treatment period were considered to be potential confounding variables and their effects were adjusted for in all multivariable models. We employed generalized estimating equations with an autoregressive correlation structure to evaluate the influence of advanced stage on QoL scores at different time points. Multivariable Cox proportional hazards models were applied to assess the prognostic effects of different scales of QoL on mortality risks. Adjusted hazard ratios (aHRs) were calculated for every 10-point increase in QoL scores, with the scores ranging from 0 to 100.

Baron and Kenny's method for identifying mediation was used to assess the possible mediating effect of QoL on the association between advanced tumor stage and patient mortality (Figure S1 illustrates the schematic processes) [26]. This approach requires all four conditions have to be met for a mediator. We applied it to our study as follows: (1) the advanced stage was significantly associated with mortality risk; (2) the advanced stage significantly affected specific QoL scales; (3) specific QoL scales significantly affect mortality risk after controlling for advanced stage; and (4) the association between advanced stage and mortality risk was higher than the same association after a specific QoL mediator was adjusted for. The scores of global health QoL, functioning QoL, QoL-C30 symptom and QoL-HN35 symptom at five time points were examined for a mediator. Mediation was measured in effect change, with the excessive effect explained by a specific QoL scale at a time point being calculated as:

$$
\text { (aHR1 - aHR2)/(aHR1 - 1), }
$$


where aHR 1 and aHR2 were the aHRs of advanced stage on patient mortality obtained, respectively, from the base and QoL-adjusted models [27-29]. The analysis was performed by using Stata version 16.0 (StataCorp, College Station, TX, USA).

\section{Results}

\subsection{Demographic and Clinical Characteristics}

This cohort included $519(76.1 \%)$ men and $163(23.9 \%)$ women with an average age of 49.4 years (Table 1 ). The majority of patients were Minnan $(80.8 \%)$, had $\leq 12$ educational years $(72.1 \%)$, were overweight $(57.9 \%)$, had no comorbidity $(\mathrm{CCI}=0 ; 72.5 \%)$, received chemotherapy $(86.2 \%)$, and were treated before 2011 (63.9\%). Higher age, being male, less educated, underweight, and treated before 2011 were significantly associated with a higher mortality risk.

Table 1. Distributions and adjusted hazard ratios (aHR) of mortality for demographic and clinical factors in nasopharyngeal cancer patient cohort $(n=682)$.

\begin{tabular}{|c|c|c|c|}
\hline Factors & No. $(\%)$ & $\mathrm{aHR}^{\mathrm{a}}$ & $(95 \% \mathrm{CI})$ \\
\hline Age, year, mean (SD) & $49.4(11.5)$ & - & - \\
\hline$\leq 40$ & $152(22.3)$ & 1.0 & - \\
\hline$>40$ & $530(77.7)$ & 1.9 & $(1.3$ to 2.9$)$ \\
\hline \multicolumn{4}{|l|}{ Gender } \\
\hline Female & $163(23.9)$ & 1.0 & - \\
\hline Male & $519(76.1)$ & 2.0 & $(1.3$ to 3.0$)$ \\
\hline \multicolumn{4}{|l|}{ Ethnicity } \\
\hline Minnan & $551(80.8)$ & 1.0 & - \\
\hline Other & $131(19.2)$ & 1.2 & (0.9 to 1.7$)$ \\
\hline \multicolumn{4}{|l|}{ Educational level, year } \\
\hline$>12$ & $190(27.9)$ & 1.0 & - \\
\hline$\leq 12$ & $491(72.1)$ & 1.6 & (1.1 to 2.3$)$ \\
\hline \multicolumn{4}{|c|}{ Body mass index $\left(\mathrm{kg} / \mathrm{m}^{2}\right)^{b}$} \\
\hline Normal (18.5-23.9) & $268(39.8)$ & 1.0 & - \\
\hline Underweight $(<18.5)$ & $16(2.4)$ & 2.4 & (1.1 to 5.1$)$ \\
\hline Overweight $(\geq 24.0)$ & $390(57.9)$ & 1.0 & (0.7 to 1.3$)$ \\
\hline \multicolumn{4}{|c|}{ Charlson comorbidity index } \\
\hline 0 & $490(72.5)$ & 1.0 & - \\
\hline$\geq 1$ & $186(27.5)$ & 1.2 & (0.9 to 1.6$)$ \\
\hline \multicolumn{4}{|l|}{ Chemotherapy } \\
\hline No & $94(13.8)$ & 1.0 & \\
\hline Yes & $588(86.2)$ & 0.7 & (0.4 to 1.2$)$ \\
\hline \multicolumn{4}{|l|}{ IMRT treatment period } \\
\hline Before 2011 & $436(63.9)$ & 1.0 & \\
\hline 2011 onward & $246(36.1)$ & 0.5 & $(0.3$ to 0.7$)$ \\
\hline \multicolumn{4}{|l|}{ Follow-up time, months } \\
\hline Mean (SD) & $90.8(50.8)$ & - & - \\
\hline Median (range) & $90.5(3.4-193.0)$ & - & - \\
\hline
\end{tabular}

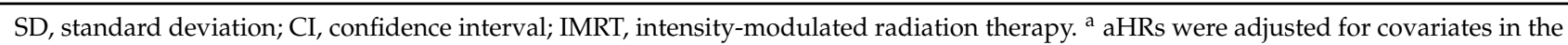
Table and AJCC stage; ${ }^{b}$ Body mass index groups were categorized according to the criterion of Health Promotion Administration, Ministry of Health and Welfare, Taiwan.

\subsection{Effects of AJCC Stage on Mortality}

Table 2 reveals that $9.5 \%, 24.5 \%, 35.8 \%$, and $30.2 \%$ of NPC patients were diagnosed with AJCC stages I to IV, respectively. Patients with stages III-IV had a significantly higher cumulative mortality rate than did patients with stages I-II (Figure $1 ; \chi^{2}=22.66, p<0.001$ ). The mortality density for NPC patients with stages I to IV was 1.8, 2.5, 3.3, and 8.2 per 
100 person-years, respectively. Compared to stage I, patients with stages III and IV had 2.5and 5.7-fold covariate-adjusted mortality risk, respectively.

Table 2. Mortality densities and adjusted hazard ratios (aHR) of mortality associated with AJCC tumor stage for nasopharyngeal cancer patients after IMRT.

\begin{tabular}{|c|c|c|c|c|c|c|c|}
\hline \multirow{2}{*}{$\begin{array}{c}\text { Group } \\
\text { Total }\end{array}$} & \multicolumn{2}{|c|}{ No. of Patients (\%) } & \multirow{2}{*}{$\begin{array}{c}\text { Years of Follow-Up } \\
5163.1\end{array}$} & \multirow{2}{*}{$\begin{array}{c}\text { No. of Deaths } \\
204\end{array}$} & \multirow{2}{*}{$\begin{array}{c}\text { Mortality Density, } \\
\text { per 100 PY } \\
3.95\end{array}$} & \multirow{2}{*}{$\frac{\mathrm{aHR}^{\mathrm{a}}}{-}$} & \multirow{2}{*}{$\begin{array}{c}(95 \% \mathrm{CI}) \\
-\end{array}$} \\
\hline & 682 & $(100.0)$ & & & & & \\
\hline AJCC stage & & & & & & & \\
\hline $\mathrm{I}$ & 65 & (9.5) & 583.0 & 10 & 1.7 & 1.0 & - \\
\hline II & 167 & $(24.5)$ & 1468.3 & 36 & 2.5 & 1.8 & $\begin{array}{c}\text { (0.8 to } \\
3.7)\end{array}$ \\
\hline III & 244 & $(35.8)$ & 1983.9 & 65 & 3.3 & 2.5 & $\begin{array}{c}\text { (1.2 to } \\
5.3)\end{array}$ \\
\hline IV & 206 & $(30.2)$ & 1127.9 & 93 & 8.2 & 5.7 & $\begin{array}{l}\text { (2.7 to } \\
12.3)\end{array}$ \\
\hline
\end{tabular}

AJCC, American Joint Committee on Cancer; IMRT, intensity-modulated radiation therapy; PY, person-years; ${ }^{a}$ aHR was adjusted for age, gender, ethnicity, educational level, body mass index, Charlson comorbidity index, chemotherapy, and IMRT treatment period.

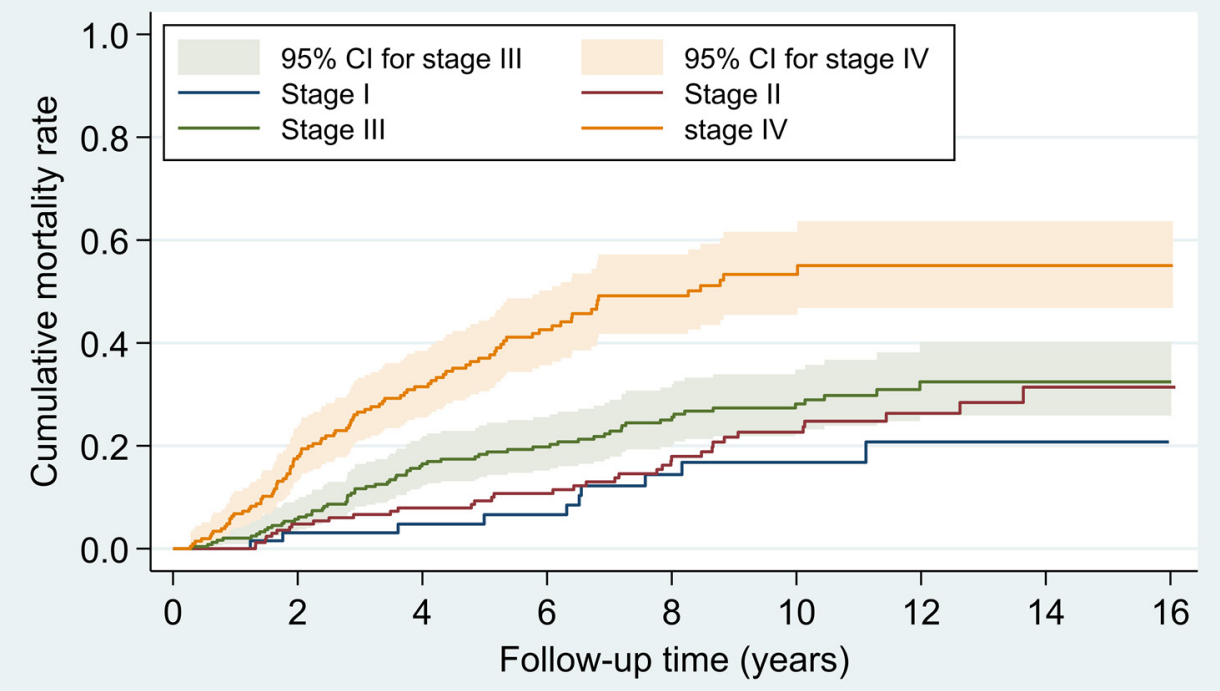

Number at risk

\begin{tabular}{|c|c|c|c|c|c|c|c|}
\hline e I & 63 & 55 & 50 & 37 & 23 & 17 & 10 \\
\hline age II 16 & 159 & 139 & 121 & 96 & 73 & 41 & 22 \\
\hline III 244 & 230 & 188 & 162 & 131 & 91 & 44 & 22 \\
\hline age IV 206 & 169 & 117 & 70 & ร? & 27 & 11 & 4 \\
\hline
\end{tabular}

Figure 1. Cumulative mortality rates of nasopharyngeal cancer patients associated with AJCC stages. Note: Cumulative mortality rates were estimated from the Kaplan-Meier estimators. Log-rank test was used to test the equality of cumulative mortality rates between AJCC stages (difference in 4 AJCC stages, $\chi^{2}=61.65, p<0.001$; difference in AJCC stage I-II versus III-IV, $\left.\chi^{2}=22.66, p<0.001\right)$. CI, confidence interval.

\subsection{Effects of AJCC Stage on QoL Score}

The distributions of QoL scores measured for global health QoL, functioning QoL, QoL-C30 symptom and QoL-HN35 symptom at the five time points are shown in Table S1. Table 3 presents the main and interaction effects of AJCC stage and QoL scores at different time points. Compared with patients with stage I-II at the time point of during IMRT, patients with stage III-IV at pre-IMRT had a significantly lower score of global health QoL and 
functioning QoL by a large magnitude and a significantly higher score of QoL-C30 symptom and QoL-HN35 symptom by a large magnitude due to the interaction effects of stage and time ( $p \leq 0.043$ for all stage $\times$ time interactions). At 3 months and 2 years post-IMRT, the advanced stage had a comparable interaction effect on the scores of global health QoL and QoL-HN35 symptom ( $p \leq 0.043$ for all stage $\times$ time interactions) and also at 3 months post-IMRT on the score of QoL-C30 symptom ( $p=0.002$ for stage $\times$ time interaction).

Compared to stage I-II patients, stage III-IV patients had a significantly lower covariate-adjusted mean score of global health QoL at pre-IMRT, 3 months and 2 years post-IMRT ( 51.6 vs. 56.6, 55.1 vs. 60.1 , and 63.4 vs. 67.8, respectively; Figure 2A) and a notably lower mean score of functioning QoL at pre-IMRT (82.8 vs. 87.1; Figure 2B). By contrast, advanced-stage patients had a significantly higher mean score of QoL-C30 symptom at pre-IMRT and 3 months post-IMRT (17.5 vs. 13.4 and 19.8 vs. 16.2, respectively; Figure 2C) and a notably higher mean score of QoL-HN35 symptom at pre-IMRT, 3 months and 2 years post-IMRT (17.4 vs. 13.0, 28.6 vs. 24.6, and 21.8 vs. 18.1, respectively; Figure 2D) than did early-stage patients.
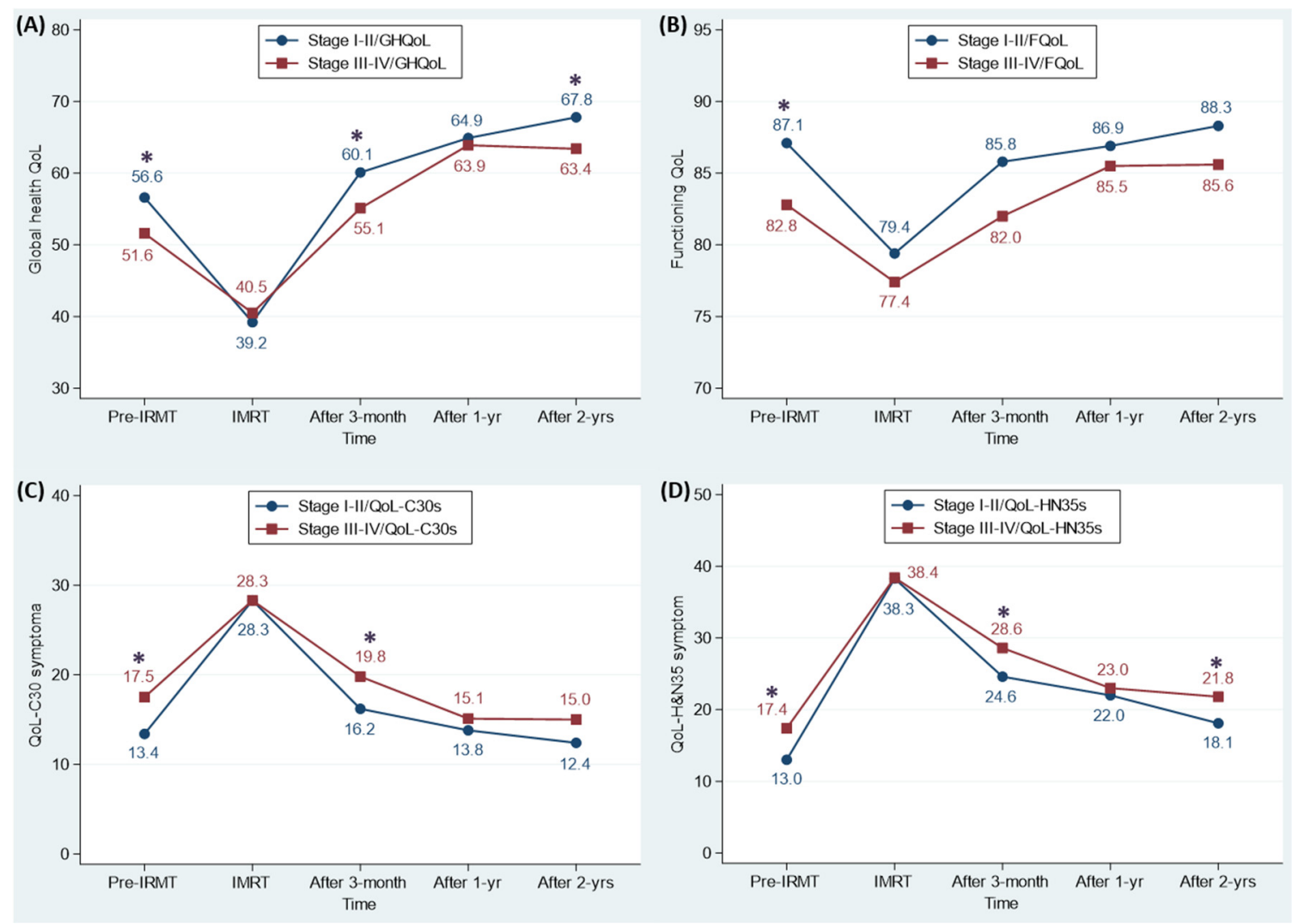

Figure 2. Distributions of adjusted quality of life (QoL) mean scores associated with AJCC stage (I-II/III-IV) at different intensity-modulated radiotherapy (IMRT)-related time points for nasopharyngeal cancer patients. (A) Global health QoL; (B) Functioning QoL; (C) QoL-C30 symptom; (D) QoL-HN35 symptom. Note: The investigated time periods included the pre-IMRT, during IMRT (40 Gy), and 3 months, 1 year, and 2 years post IMRT. The QoL scores were adjusted for age, gender, ethnicity, educational level, body mass index, Charlson comorbidity index, chemotherapy, and IMRT treatment period. * denotes significant difference in the adjusted QoL mean scores between AJCC stage I-II and III-IV. GHQoL, Global Health QoL, FQoL, Functioning QoL, QoL-C30 symptom, and QoL-HN35 symptom denoted the average QoL scores obtained from the EORTC QLQ-C30 global QoL scale, 5 functional scales, and 9 symptom scales/items, and EORTC QLQ-HN35 18 symptom scales/items, respectively. IMRT, intensity-modulated radiotherapy; yrs, years; EORTC, European organization for research and treatment of cancer; QoL-C30, the core QoL questionnaire of EORTC; QoL-HN35, the head and neck cancer-specific QoL questionnaire module of EORTC. 
Table 3. Main and interaction effect of AJCC stage and quality of life (QoL) scores at different time points.

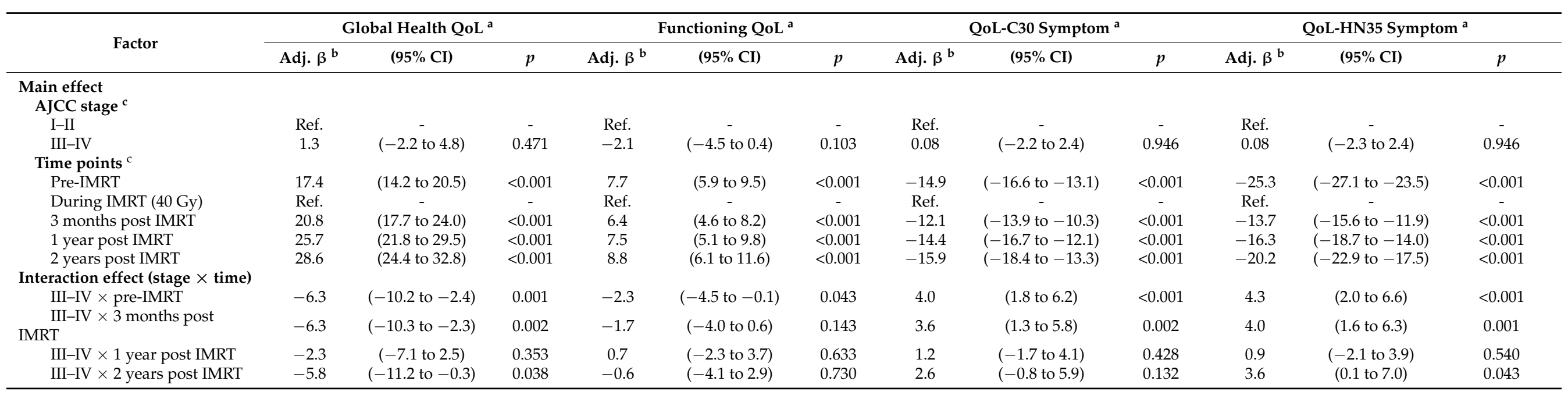

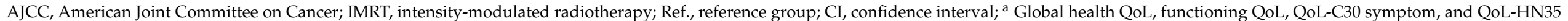

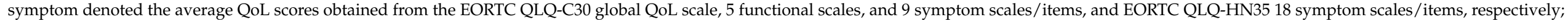

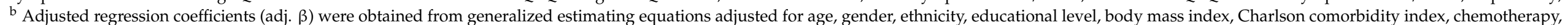
and IMRT treatment period; ${ }^{\mathrm{c}}$ Patients with stage I-II and at the time pint of during IMRT (40 Gy) were the reference groups. 


\subsection{Effects of QoL Score on Mortality}

Table 4 presents the covariates and AJCC stage-adjusted association between various QoL scores and mortality at the five time points. Patients with a higher level of global health QoL at pre-IMRT and a higher score of global health and functioning QoL at 1 and 2 years post-IMRT exhibited a lower mortality risk ( $\mathrm{aHR}=0.79-0.92$ per 10 -point increase; all $p<0.05)$. By contrast, patients having a higher score of QoL-C30 symptom and QoL-HN35 symptom at the abovementioned time points revealed a higher mortality risk (aHR $=1.15-1.34$ per 10-point increase; all $p<0.05)$.

Table 4. Adjusted hazard ratios (aHR) of mortality associated with continuous quality of life (QoL) scores at different time points of IMRT.

\begin{tabular}{|c|c|c|c|c|c|c|c|c|c|c|}
\hline \multirow{2}{*}{${ }^{\mathrm{b}}$ QoL Scores } & \multicolumn{2}{|c|}{ Pre-IMRT } & \multicolumn{2}{|c|}{ During IMRT (40 Gy) } & \multicolumn{2}{|c|}{3 Months Post IMRT } & \multicolumn{2}{|c|}{1 Year Post IMRT } & \multicolumn{2}{|c|}{2 Years Post IMRT } \\
\hline & $\mathrm{aHR}^{\mathrm{a}}$ & $(95 \% \mathrm{CI})$ & $\mathrm{aHR}^{\mathrm{a}}$ & (95\% CI) & $\mathrm{aHR}^{\mathrm{a}}$ & $(95 \% \mathrm{CI})$ & $\mathrm{aHR}^{\mathrm{a}}$ & (95\% CI) & $\mathrm{aHR}^{\mathrm{a}}$ & $(95 \% \mathrm{CI})$ \\
\hline Global health QoL & $0.92 *$ & $(0.86$ to 0.99$)$ & 0.94 & (0.87 to 1.02$)$ & 0.97 & (0.89 to 1.05$)$ & $0.88 *$ & (0.80 to 0.97$)$ & $0.79 *$ & (0.69 to 0.91$)$ \\
\hline Functioning QoL & 0.90 & (0.82 to 1.00$)$ & 0.97 & (0.88 to 1.06$)$ & 0.93 & (0.83 to 1.03$)$ & $0.85^{*}$ & (0.76 to 0.96$)$ & $0.80 *$ & (0.67 to 0.96$)$ \\
\hline QoL-C30 symptom & $1.15^{*}$ & (1.03 to 1.28$)$ & 1.00 & (0.90 to 1.11$)$ & 1.08 & (0.97 to 1.21$)$ & $1.19 *$ & (1.05 to 1.35$)$ & $1.34 *$ & (1.11 to 1.63$)$ \\
\hline QoL-HN35 symptom & $1.17^{*}$ & (1.05 to 1.31$)$ & 1.02 & (0.92 to 1.12$)$ & 1.02 & (0.91 to 1.15$)$ & $1.18^{*}$ & (1.04 to 1.34$)$ & $1.34 *$ & (1.11 to 1.61$)$ \\
\hline
\end{tabular}

IMRT: intensity modulated radiotherapy; ${ }^{*} p<0.05 ;{ }^{a}$ aHRs were displayed as the risks for every 10-point increase in QoL scores and were adjusted for age, gender, ethnicity, educational level, body mass index, Charlson comorbidity index, chemotherapy, and IMRT treatment period, as well as AJCC stage.; ${ }^{b}$ Global health QoL, functioning QoL, QoL-C30 symptom, and QoL-HN35 symptom denoted the average QoL scores obtained from the EORTC QLQ-C30 global QoL scale, 5 functional scales, and 9 symptom scales/items, and EORTC QLQ-HN35 18 symptom scales/items, respectively. EORTC, European organization for research and treatment of cancer; QoL-C30, the core QoL questionnaire of EORTC; QoL-HN35, the head and neck cancer-specific QoL questionnaire module of EORTC.

\subsection{Effect Changes Associated with QoL Score Mediation}

According to the four requirements of Baron and Kenny's approach for a mediator, global health QoL, QoL-C30 symptom and QoL-HN35 symptom at pre-IMRT and global health QoL and QoL-HN35 symptom at 2 years post-IMRT exhibited an intermediated effect on the association between advanced stage and high mortality risk (Figure 3). Compared with stage I-II patients, stage III-IV patients had a 2.26-fold covariate-adjusted mortality risk (Table 5, base model). In the base model that was additionally adjusted for global health QoL, QoL-C30 symptom and QoL-HN35 symptom at pre-IMRT, the observed excessive risk was reduced to 2.19-, 2.14-, and 2.14-fold, respectively, with effect changes of $5.8 \%, 9.8 \%$ and $9.3 \%$. By contrast, the scores of global health QoL and QoL-HN35 symptom at 2 years post-IMRT explained $49.4 \%$ and $39.4 \%$ of the excessive effect of advanced stage on mortality risk (the aHRs reduced to 1.64 and 1.76, respectively, when the base model was additionally adjusted for the two QoL scores).

Table 5. Adjusted hazard ratios (aHR) of mortality and effect changes in mortality risks associated with AJCC stage and quality of life (QoL) scores at different time points of IMRT.

\begin{tabular}{|c|c|c|c|c|}
\hline \multirow{2}{*}{ Models/Variables } & \multicolumn{3}{|c|}{ Stages III-IV vs. I-II } & \multirow{2}{*}{$\mathrm{EC}^{\mathrm{a}}$} \\
\hline & aHR & $(95 \%$ CI $)$ & $p$ Value & \\
\hline Base model $^{b}$ & 2.26 & (1.56 to 3.27$)$ & $<0.001$ & Ref. \\
\hline \multicolumn{5}{|l|}{$\begin{array}{l}\text { Base model + QoL scale }{ }^{c, d} \\
\text { Pre-IMRT }\end{array}$} \\
\hline Global health QoL & 2.19 & (1.51 to 3.17$)$ & $<0.001$ & $5.8 \%$ \\
\hline QoL-C30 symptom & 2.14 & (1.47 to 3.10$)$ & $<0.001$ & $9.8 \%$ \\
\hline QoL-HN35 symptom & 2.14 & (1.48 to 3.10$)$ & $<0.001$ & $9.3 \%$ \\
\hline \multicolumn{5}{|l|}{2 years post IMRT } \\
\hline Global health QoL & 1.64 & (0.92 to 2.90$)$ & 0.091 & $49.4 \%$ \\
\hline QoL-HN35 symptom & 1.76 & (0.99 to 3.12$)$ & 0.052 & $39.4 \%$ \\
\hline
\end{tabular}

AJCC, American Joint Committee on Cancer; IMRT, intensity-modulated radiotherapy; Ref., reference group; ${ }^{\text {a }}$ Effect change (EC) was the excessive effect explained by a specific QoL scale at a time period. It was calculated as: [(Base model aHR - QoL-adjusted aHR)/(Base model aHR - 1)] $\times 100{ }^{\mathrm{b}}$ aHR in the base model was adjusted for age, gender, ethnicity, educational level, body mass index, Charlson comorbidity index, chemotherapy, and IMRT treatment period; ${ }^{c}$ aHRs were obtained from the base model additionally adjusted for a specific QoL scale.; ${ }^{\mathrm{d}}$ Global health QoL, functioning QoL, QoL-C30 symptom, and QoL-HN35 symptom denoted the average QoL scores obtained from the EORTC QLQ-C30 global QoL scale, 5 functional scales, and 9 symptom scales/items, and EORTC QLQ-HN35 18 symptom scales/items, respectively. EORTC, European organization for research and treatment of cancer; QoL-C30, the core QoL questionnaire of EORTC; QoL-HN35, the head and neck cancer-specific QoL questionnaire module of EORTC. 


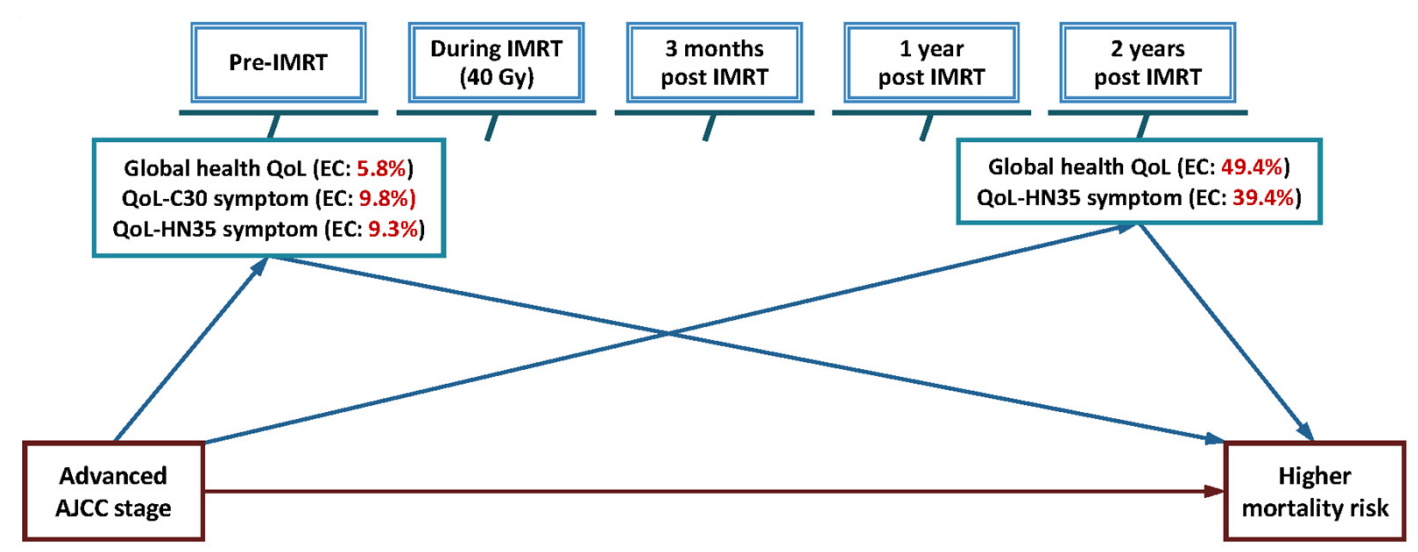

Figure 3. The intermediated paths of quality of life (QoL) scales at different intensity-modulated radiation therapy (IMRT)related time points on the association between advanced AJCC stage (III-IV) and higher mortality risk in nasopharyngeal cancer patient follow-up cohort (intermediated path: Advanced AJCC stage to QoL score to Mortality risk). Note: Effect change (EC) was the excessive effect explained by a specific QoL scale at a time period. Global health QoL, QoL-C30 symptom, and QoL-HN35 symptom denoted the average QoL scores obtained from the EORTC QLQ-C30 global QoL scale, 9 symptom scales/items, and EORTC QLQ-HN35 18 symptom scales/items, respectively. AJCC, American Joint Committee on Cancer; EORTC, European organization for research and treatment of cancer; QoL-C30, the core QoL questionnaire of EORTC; QoL-HN35, the head and neck cancer-specific QoL questionnaire module of EORTC.

\section{Discussion}

As far as we know, the study is the first time to demonstrate that QoL acts as a mediator between cancer stage and long-term mortality of cancer patients. For NPC patients, this longitudinal study presents comprehensive findings to demonstrate that AJCC stages III-IV influence patients' QoL levels at different IMRT-associated time points and that specific QoL scales influence patient mortality. Although the advanced cancer stage in NPC patients significantly predicted high mortality risk, the effects were partially mediated by specific QoL scales at pre-IMRT and 2 years post-IMRT.

NPC patients often exhibit clear debilitating issues of swallowing or hearing and have psycho-social problems related to loss of daily function after treatments, which makes QoL an important outcome evaluation of medical care for this type of cancer [30]. A 14-study systematic assessment on xerostomia-related QoL for IMRT-treated NPC patients revealed that the worst QoL measured in multidimensional scales occurred during or at the end of treatment, but a gradual recovery was observed at 1-2 years after IMRT [23]. Similar findings were recognized in this large-scale NPC patient cohort, in that advanced- and earlystage patients both had worse QoL levels in terms of global health QoL, functioning QoL, QoL-C30 symptom, and QoL-HN35 symptom during IMRT (Figure 2). Our investigation also identified that these four QoL levels gradually improved; however, 3 months postIMRT, advanced-stage patients had worse global health QoL, QoL-C30 symptom, and QoL-HN35 symptom than early patients and at 2 years post-IMRT they maintained poor global health QoL and QoL-HN35 symptom. The five time trajectory distributions for QoL and the interplay effect of cancer stage and time on the four QoL scales provide empirical information for clinicians to understand the association between IMRT and QoL in advanced- and early-stage NPC patients.

The added value of QoL has been paid ample attention in clinical therapy due to several QoL scales showing independent predictive capability for cancer patient survival [6-11]. A meta-analysis of individual patient data from 30 EORTC clinical trials for 11 different cancers suggested that QoL information can help to predict survival for cancer patients [6]. A collective study assessing 17 Canadian randomized controlled trials for eight carcinoma sites supported this argument and indicated that baseline QLQ-C30 QoL scores offer prognostic information in addition to the data from demographic and clinical variables [7]. In a 
longitudinal study for head and neck carcinoma, specific pretreatment QoL measures, such as dyspnea and appetite loss, were found to link to overall survival [8]. In prospective investigations for NPC patients treated with three-dimensional conformal radiotherapy or IMRT, QoL scales measured at pretreatment and 1 year after treatment have been recognized to have a prognostic value for survival [9-11]. Using a comprehensive QoL assessment at five IMRT-related time points, our study further identified that the predictive capability of QoL for NPC patient survival was not restricted to pretreatment and 1 year after IMRT but was extended to 2 years after IMRT, with the strongest prognostic effect of QoL, in fact, being observed at 2 years post-IMRT.

Clinical studies reported that a high proportion of NPC patients were confirmed as AJCC stages III-IV at diagnosis [16-18]. Because cancer stage is an unchangeable factor and closely linked to a high mortality [3,4], this highlights the issue of how to prolong survival for advanced-stage NPC patients. QoL in recent studies was recommended as a vital outcome measure in clinical decision-making, especially for advanced-stage cancer patients $[6,7,31,32]$. Cancer studies using pooled data have indicated that the inclusion of physical functioning, pain and appetite-loss-related QoL in the model with demographic and clinical variables can increase the predictive accuracy by $6 \%$ for overall survival of eleven cancers in Europe [6], and the added prognostic value of global health, dyspnea and appetite-loss-related QoL for overall survival of eight cancers was 5\% in Canada [7]. Clinical prospective investigations also found that the improvement of QoL within 3 months of treatment was significantly associated with a reduced risk of mortality in colorectal, prostate, and pancreatic cancer patients, even if the related QoL scales were different [33-35].

In this study with multiple evaluations for multiple QoL scales over five IMRT-related time points, our findings indicated that $5.8-9.8 \%$ of mortality risk for advanced-stage NPC patients was mediated by global health QoL, QoL-C30 symptom, and QoL-HN35 symptom at pre-IMRT. This implies that improvement in these three QoL levels before IMRT treatment is the first step that physicians and families need to help the patients. Our study also revealed that the levels of global health QoL and QoL-HN35 symptom at 2 years post-IMRT mediated a substantial proportion of mortality risk for NPC patients with stages III-IV (39.4-49.4\%). This emphasizes that the improvement of global health QoL and QoL-HN35 symptom should continue up to 2 years after IMRT and that this time point could be regarded as a vital QoL checkpoint for monitoring patient survival or tailoring QoL-dependent medical support.

A question in this study is why the mediating effect of QoL on the relationship between NPC stage and mortality significantly manifested 2 years post-IMRT but not at earlier time points. Before IMRT, 3 months post-IMRT, and 2 years post-IMRT, patients with advanced NPC exhibited lower global health QoL and higher QoL-HN35 symptom than did patients with early-stage NPC. However, only the QoL measurements taken pre-IMRT and 2-years post-IMRT were associated with mortality, and a greater effect was observed 2-years post-IMRT than pre-IMRT (Table 4). Moreover, the four QoL measurements taken 1-year post-IMRT were related to mortality, but all QoL scores were nonsignificant between patients in the early and advanced stages (Figure 2). According to the rule established by Baron and Kenny [26], all four mediation conditions have to be met for a variable to be a mediator. Thus, the main mediating effect of QoL manifested 2 years post-IMRT. In a clinical setting, radiation-related late toxicity is a gradual process that is exacerbated with time. In a previous report, the severity of IMRT-related late toxicity was associated with the QoL outcomes of NPC survivors [36]. Therefore, the mortality-mediating effect of QoL 2 years post-IMRT may reflect the late toxicity effect of radiation-related treatment among patients with NPC.

Two strategies for medical treatment and care can improve global health QoL and QoLHN35 symptom 2 years post-IMRT. The first strategy involves using specific radiotherapy to minimize radiation-related late toxicity. Suggested treatments include the administration of particle or proton therapy, implementation of response-adapted treatment plans following 
neoadjuvant chemotherapy or during the course of radiotherapy, and reduction in elective nodal volume in certain cases [37]. The second strategy entails enhancing QoL through specific interventions. Recommended interventions include the provision of swallowing function training and oral health care training to reduce the severity of dysphagia [38,39], the substitution of saliva for mouthwash to reduce xerostomia [40], implementation of regular home nursing interventions to improve global health [41], implementation of nutrition support and head-and-neck rehabilitation exercise to ameliorate fatigue [42], implementation of psychological interventions (particularly cognitive behavioral therapy) to mitigate depression or anxiety [43], and implementation of transdisciplinary geriatric and palliative care interventions to enhance the QoL of older adults with cancer [44].

This study has several limitations: first; certain clinical and biological mechanisms may be involved in the association between advanced-stage NPC and high mortality risk, but we only investigated the intermediated effects of QoL from pre-IMRT to within 2 years after IMRT. Second, we did not consider the Epstein-Barr virus because the virus DNA detection data were not fully available in the cohort. Third, the difference in $4 \mathrm{QoL}$ scores for the group comparison at a significant level ranged from 3.6 to 5.0 units, their clinical significances need to be further investigated. Last, $26 \%$ of NPC patients were lost to follow-up at 2-years, which may introduce selection bias in the results. Since the distributions of demographic and clinical factors, such as age, sex, ethnicity, body mass index, Charlson comorbidity index, chemotherapy, and IMRT treatment period for the remained and loss patients were comparable, if the bias exists, the degree would be limited. Alternatively, a major strength of this study is that it is the first to elucidate the associations across advanced-stage cancer between multiple health-associated QoL scales over five IMRT-related time points and long-term mortality among NPC patients. Furthermore, the QoL data evaluated at multiple IMRT-associated time points offer clinicians comprehensive information for QoL-dependent medical decisions and care management at the appropriate treatment time.

\section{Conclusions}

We concluded advanced cancer stage correlates with a long-term high mortality in NPC patients treated with IMRT and the association is partially intermediated by QoL at pre-IMRT and 2 years post-IMRT. Therefore, QoL-HN35 symptom and global health QoLdependent medical support and care should be focused and tailored at 2 years post-IMRT.

Supplementary Materials: The following are available online at https:/ / www.mdpi.com/article/ 10.3390 / cancers13205063/s1, Figure S1: The processes of assessing quality of life (QoL) at different intensity-modulated radiation therapy (IMRT)-related time periods as a possible mediator on the relationship between advanced AJCC tumor stage (III-IV) and higher mortality risk in nasopharyngeal cancer patient follow-up cohort, Table S1: Distributions of quality of life (QoL) scores of EORTC QLQ-C30 and EORTC QLQ-HN35 at different IMRT-related time points for nasopharyngeal cancer patients.

Author Contributions: Conceptualization, K.-C.L., F.-M.F. and C.-H.L.; methodology, K.-C.L., F.-M.F. and C.-H.L. and W.-T.L.; validation, H.-C.C., C.-Y.C., Y.-T.L. and M.-H.T.; formal analysis, K.-C.L. and C.-H.L.; investigation, F.-M.F. and T.-F.L.; resources, H.-C.C., C.-Y.C., Y.-T.L., M.-H.T., Y.-Y.S., C.-H.Y., C.-C.L., T.-L.H. and S.-H.L.; data curation, C.-H.L.; writing-original draft preparation, K.-C.L.; writing-review and editing, F.-M.F. and C.-H.L.; supervision, H.-C.C., F.-M.F. and C.-H.L.; project administration, K.-C.L., F.-M.F. and C.-H.L.; funding acquisition, F.-M.F. All authors have read and agreed to the published version of the manuscript.

Funding: Chang Gung Memorial Hospital, Support Grant No. CMRPG8F1201-3, CMRPG8J1031-2, and CMRPG8K1601, and partially supported by the grant of the Research Center for Environmental Medicine, Kaohsiung Medical University (KMU-TC108A01) from the Featured Areas Research Center Program within the framework of the Higher Education Sprout Project by the Taiwan Ministry of Education. 
Institutional Review Board Statement: This research was approved by the Chang-Gung Medical Foundation Institutional Review Board (No. 202100354B0).

Informed Consent Statement: Informed consent was obtained from all subjects involved in the study.

Data Availability Statement: The data presented in this study are available on request from the corresponding author. The data are not publicly available due to ethical restrictions.

Conflicts of Interest: The authors declare no conflict of interest.

\section{References}

1. Chen, Y.; Chan, A.T.C.; Le, Q.-T.; Blanchard, P.; Sun, Y.; Ma, J. Nasopharyngeal carcinoma. Lancet 2019, 394, 64-80. [CrossRef]

2. Lee, A.W.; Ng, W.T.; Chan, L.L.; Hung, W.M.; Chan, C.C.; Sze, H.C.; Chan, O.S.H.; Chang, A.T.; Yeung, R.M. Evolution of treatment for nasopharyngeal cancer-Success and setback in the intensity-modulated radiotherapy era. Radiother. Oncol. 2014, 110, 377-384. [CrossRef] [PubMed]

3. Kanno, M.; Narita, N.; Fujimoto, Y.; Wakisaka, N.; Yoshizaki, T.; Kodaira, T.; Makita, C.; Sato, Y.; Yamazaki, K.; Wakaoka, T.; et al. Third Epidemiological Analysis of Nasopharyngeal Carcinoma in the Central Region of Japan from 2006 to 2015. Cancers 2019, 11, 1180. [CrossRef] [PubMed]

4. Ruuskanen, M.; Grenman, R.; Leivo, I.; Vahlberg, T.; Mäkitie, A.; Saarilahti, K.; Wigren, T.; Korpela, M.; Voutilainen, L.; Koivunen, P.; et al. Outcome of nasopharyngeal carcinoma in Finland: A nationwide study. Acta Oncol. 2018, 57, 251-256. [CrossRef] [PubMed]

5. Sitlinger, A.; Zafar, S.Y. Health-Related Quality of Life: The impact on morbidity and mortality. Surg. Oncol. Clin. N. Am. 2018, 27, 675-684. [CrossRef]

6. Quinten, C.; Coens, C.; Mauer, M.; Comte, S.; Sprangers, M.A.; Cleeland, C.; Osoba, D.; Bjordal, K.; Bottomley, A. Baseline quality of life as a prognostic indicator of survival: A meta-analysis of individual patient data from EORTC clinical trials. Lancet Oncol. 2009, 10, 865-871. [CrossRef]

7. Ediebah, D.E.; Quinten, C.; Coens, C.; Ringash, J.; Dancey, J.; Zikos, E.; Gotay, C.; Brundage, M.; Tu, D.; Flechtner, H.; et al. Quality of life as a prognostic indicator of survival: A pooled analysis of individual patient data from Canadian cancer trials group clinical trials. Cancer 2018, 124, 3409-3416. [CrossRef]

8. Yang, C.J.; Roh, J.-L.; Kim, M.-J.; Lee, S.-W.; Kim, S.-B.; Choi, S.-H.; Nam, S.Y.; Kim, S.Y. Pretreatment quality of life as a prognostic factor for early survival and functional outcomes in patients with head and neck cancer. Qual. Life Res. 2016, 25, 165-174. [CrossRef] [PubMed]

9. $\quad$ Fang, F.-M.; Tsai, W.-L.; Chien, C.-Y.; Chen, H.-C.; Hsu, H.-C.; Huang, T.-L.; Lee, T.-F.; Huang, H.-Y.; Lee, C.-H. Pretreatment Quality of Life as a Predictor of Distant Metastasis and Survival for Patients with Nasopharyngeal Carcinoma. J. Clin. Oncol. 2010, 28, 4384-4389. [CrossRef] [PubMed]

10. Tsai, W.-L.; Chien, C.-Y.; Huang, H.-Y.; Liao, K.-C.; Fang, F.-M. Prognostic value of quality of life measured after treatment on subsequent survival in patients with nasopharyngeal carcinoma. Qual. Life Res. 2013, 22, 715-723. [CrossRef]

11. Guo, S.-S.; Hu, W.; Chen, Q.-Y.; Li, J.-M.; Zhu, S.-H.; He, Y.; Li, J.-W.; Xia, L.; Ji, L.; Lin, C.-Y.; et al. Pretreatment quality of life as a predictor of survival for patients with nasopharyngeal carcinoma treated with IMRT. BMC Cancer 2018, 18, 1-9. [CrossRef]

12. Huang, T.-L.; Chien, C.-Y.; Tsai, W.-L.; Liao, K.-C.; Chou, S.-Y.; Lin, H.-C.; Luo, S.D.; Lee, T.-F.; Lee, C.-H.; Fang, F.-M. Long-term late toxicities and quality of life for survivors of nasopharyngeal carcinoma treated with intensity-modulated radiotherapy versus non-intensity-modulated radiotherapy. Head Neck 2016, 38, E1026-E1032. [CrossRef] [PubMed]

13. McDowell, L.J.; Rock, K.; Xu, W.; Chan, B.; Waldron, J.; Lu, L.; Ezzat, S.; Pothier, D.; Bernstein, L.; So, N.; et al. Long-Term Late Toxicity, Quality of Life, and Emotional Distress in Patients with Nasopharyngeal Carcinoma Treated with Intensity Modulated Radiation Therapy. Int. J. Radiat. Oncol. 2018, 102, 340-352. [CrossRef] [PubMed]

14. Cengiz, M.; Özyar, E.; Esassolak, M.; Altun, M.; Akmansu, M.; Şen, M.; Uzel, O.; Yavuz, A.; Dalmaz, G.; Uzal, C.; et al. Assessment of quality of life of nasopharyngeal carcinoma patients with EORTC QLQ-C30 and H\&N-35 modules. Int. J. Radiat. Oncol. 2005, 63, 1347-1353. [CrossRef]

15. Hong, J.; Tian, J.; Han, Q.; Ni, Q. Quality of Life of Nasopharyngeal Cancer Survivors in China. Curr. Oncol. 2015, 22, 142-147. [CrossRef] [PubMed]

16. Xu, L.; Yao, J.-J.; Zhou, G.-Q.; Zhang, W.; Liu, G.-L.; Liu, L.-Z.; Ma, J.; Sun, Y. The Impact of Clinical Stage on Radiation Doses to Organs at Risk Following Intensity-modulated Radiotherapy in Nasopharyngeal Carcinoma: A Prospective Analysis. J. Cancer 2016, 7, 2157-2164. [CrossRef]

17. Haleshappa, R.A.; Thanky, A.H.; Kuntegowdanahalli, L.; Kanakasetty, G.B.; Dasappa, L.; Jacob, L. Epidemiology and outcomes of nasopharyngeal carcinoma: Experience from a regional cancer center in Southern India. South Asian J. Cancer 2017, 6, 122-124. [CrossRef]

18. Abdullah, M.M.; Foo, Y.C.; Yap, B.K.; Lee, C.M.L.; Hoo, L.P.; Lim, T.O. Retrospective Analysis of Cancer Care Performance and Survival Outcome for Nasopharyngeal Carcinoma at a leading Cancer Treatment Centre in Malaysia 2008-2012. Asian Pac. J. Cancer Prev. 2019, 20, 1701-1708. [CrossRef] 
19. Amin, M.B.; Greene, F.L.; Edge, S.B.; Compton, C.C.; Gershenwald, J.E.; Brookland, R.K.; Meyer, L.; Gress, D.M.; Byrd, D.R.; Winchester, D.P. The Eighth Edition AJCC Cancer Staging Manual: Continuing to build a bridge from a population-based to a more "personalized" approach to cancer staging. CA Cancer J. Clin. 2017, 67, 93-99. [CrossRef]

20. Fang, F.; Huang, T.; Lin, Y.; Chien, C.; Chuang, H.; Luo, S.; Lin, H.; Lin, Y.; Li, S.; Liao, K.; et al. Concurrent chemoradiotherapy by simultaneously integrated boost volumetric-modulated arc therapy for nasopharyngeal carcinoma-toxicity/quality of life and survival. Head Neck 2019, 41, 1282-1289. [CrossRef]

21. Chie, W.; Hong, R.; Lai, C.; Ting, L.; Hsu, M. Quality of life in patients of nasopharyngeal carcinoma: Validation of the Taiwan Chinese version of the EORTC QLQ-C30 and the EORTC QLQ-H\&N35. Qual. Life Res. 2003, 12, 93-98. [CrossRef] [PubMed]

22. Fayers, P.; Aaronson, N.K.; Bjordal, K.; Groenvold, M.; Curran, D.; Bottomley, A. EORTC QLQ-C30 Scoring Manual, 3rd ed.; European Organisation for Research and Treatment of Cancer: Brussel, Belgium, 2001.

23. Bian, X.; Song, T.; Wu, S. Outcomes of xerostomia-related quality of life for nasopharyngeal carcinoma treated by IMRT: Based on the EORTC QLQ-C30 and H\&N35 questionnaires. Expert Rev. Anticancer Ther. 2015, 15, 109-119. [CrossRef] [PubMed]

24. Chung, W.-S.; Ho, F.-M.; Cheng, N.-C.; Lee, M.-C.; Yeh, C.-J. BMI and all-cause mortality among middle-aged and older adults in Taiwan: A population-based cohort study. Public Health Nutr. 2015, 18, 1839-1846. [CrossRef] [PubMed]

25. Deyo, R.A.; Cherkin, D.C.; Ciol, M.A. Adapting a clinical comorbidity index for use with ICD-9-CM administrative databases. J. Clin. Epidemiol. 1992, 45, 613-619. [CrossRef]

26. Baron, R.M.; Kenny, D.A. The moderator-mediator variable distinction in social psychological research: Conceptual, strategic, and statistical considerations. J. Pers. Soc. Psychol. 1986, 51, 1173-1182. [CrossRef] [PubMed]

27. Jung, S.Y.; Rosenzweig, M.; Linkov, F.; Brufsky, A.; Weissfeld, J.L.; Sereika, S.M. Comorbidity as a Mediator of Survival Disparity Between Younger and Older Women Diagnosed with Metastatic Breast Cancer. Hypertension 2012, 59, 205-211. [CrossRef]

28. Lee, C.-Y.; Lin, W.-T.; Tsai, S.; Hung, Y.-C.; Wu, P.-W.; Yang, Y.-C.; Chan, T.-F.; Huang, H.-L.; Weng, Y.-L.; Chiu, Y.-W.; et al. Association of Parental Overweight and Cardiometabolic Diseases and Pediatric Adiposity and Lifestyle Factors with Cardiovascular Risk Factor Clustering in Adolescents. Nutrients 2016, 8, 567. [CrossRef]

29. Lin, W.-T.; Chan, T.-F.; Huang, H.-L.; Lee, C.-Y.; Tsai, S.; Wu, P.-W.; Yang, Y.-C.; Wang, T.-N.; Lee, C.-H. Fructose-Rich Beverage Intake and Central Adiposity, Uric Acid, and Pediatric Insulin Resistance. J. Pediatr. 2016, 171, 90-96.e1. [CrossRef]

30. Terrell, J.E.; Nanavati, K.; Esclamado, R.M.; Bradford, C.R.; Wolf, G.T. Health impact of head and neck cancer. Otolaryngol. Neck Surg. 1999, 120, 852-859. [CrossRef]

31. Bottomley, A.; Therasse, P.; Piccart, M.; Efficace, F.; Coens, C.; Gotay, C.; Welnicka-Jaskiewicz, M.; Mauriac, L.; Dyczka, J.; Cufer, T.; et al. Health-related quality of life in survivors of locally advanced breast cancer: An international randomised controlled phase III trial. Lancet Oncol. 2005, 6, 287-294. [CrossRef]

32. Barton, D.L.; Thanarajasingam, G.; Sloan, J.A.; Diekmann, B.; Fuloria, J.; Kottschade, L.A.; Lyss, A.P.; Jaslowski, A.J.; Mazurczak, M.A.; Blair, S.C.; et al. Phase III double-blind, placebo-controlled study of gabapentin for the prevention of delayed chemotherapyinduced nausea and vomiting in patients receiving highly emetogenic chemotherapy, NCCTG N08C3 (Alliance). Cancer 2014, 120, 3575-3583. [CrossRef] [PubMed]

33. Braun, D.P.; Gupta, D.; Grutsch, J.F.; Staren, E.D. Can changes in health related quality of life scores predict survival in stages III and IV colorectal cancer? Health Qual. Life Outcomes 2011, 9, 62. [CrossRef] [PubMed]

34. Gupta, D.; Braun, D.P.; Staren, E.D. Prognostic value of changes in quality of life scores in prostate cancer. BMC Urol. 2013, 13, 32. [CrossRef] [PubMed]

35. Braun, D.P.; Gupta, D.; Staren, E.D. Longitudinal Health-Related Quality of Life Assessment Implications for Prognosis in Stage IV Pancreatic Cancer. Pancreas 2013, 42, 254-259. [CrossRef] [PubMed]

36. Tsai, W.-L.; Huang, T.-L.; Liao, K.-C.; Chuang, H.-C.; Lin, Y.-T.; Lee, T.-F.; Huang, H.-Y.; Fang, F.-M. Impact of late toxicities on quality of life for survivors of nasopharyngeal carcinoma. BMC Cancer 2014, 14, 1-8. [CrossRef] [PubMed]

37. McDowell, L.; Corry, J.; Ringash, J.; Rischin, D. Quality of Life, Toxicity and Unmet Needs in Nasopharyngeal Cancer Survivors. Front. Oncol. 2020, 10, 930. [CrossRef]

38. Hajdú, S.F.; Wessel, I.; Dalton, S.O.; Eskildsen, S.J.; Johansen, C. Swallowing Exercise During Head and Neck Cancer Treatment: Results of a Randomized Trial. Dysphagia 2021, 1-14. [CrossRef]

39. Morais, M.O.; Martins, A.F.L.; De Jesus, A.P.G.; Neto, S.S.D.S.; Da Costa, A.W.F.; Pereira, C.H.; Oton-Leite, A.F.; De Freitas, N.M.A.; Leles, C.R.; Mendonça, E.F. A prospective study on oral adverse effects in head and neck cancer patients submitted to a preventive oral care protocol. Support. Care Cancer 2020, 28, 4263-4273. [CrossRef]

40. Marimuthu, D.; Han, K.M.; Mohamad, M.S.F.; Azman, M. Saliva substitute mouthwash in nasopharyngeal cancer survivors with xerostomia: A randomized controlled trial. Clin. Oral Investig. 2020, 25, 3105-3115. [CrossRef]

41. Shi, R.-C.; Meng, A.-F.; Zhou, W.-L.; Yu, X.-Y.; Huang, X.-E.; Ji, A.-J.; Chen, L. Effects of Home Nursing Intervention on the Quality of Life of Patients with Nasopharyngeal Carcinoma after Radiotherapy and Chemotherapy. Asian Pac. J. Cancer Prev. 2015, 16, 7117-7121. [CrossRef]

42. Su, D.; He, Y.; Chen, L.; Li, J.; Zhang, L.; Chen, L.; Li, W.; Hu, W.; Li, C.; Fan, Y. Nutrition counseling combined with head and neck rehabilitation exercises can enhance outcomes among nasopharyngeal carcinoma patients in southern China: A prospective study in an epidemic area. Ann. Palliat. Med. 2020, 9, 1152-1163. [CrossRef] [PubMed] 
43. Liu, F.; Fu, S.-N.; Chen, Y.-Z.; Yan, O.-Y.; Tong, F.; Peng, W.-L.; Zou, R.; Wen, M.-N.; Jiang, L.; Ma, H.-Z.; et al. Effects of Cognitive Behavioral Therapy for Depression and Anxiety, Response Rates and Adverse Events in Patients with Locoregional Advanced Nasopharyngeal Carcinoma. Integr. Cancer Ther. 2021, 20. [CrossRef] [PubMed]

44. Nipp, R.D.; Temel, B.; Fuh, C.-X.; Kay, P.; Landay, S.; Lage, D.; Franco-Garcia, E.; Scott, E.; Stevens, E.; O’Malley, T.; et al. Pilot Randomized Trial of a Transdisciplinary Geriatric and Palliative Care Intervention for Older Adults with Cancer. J. Natl. Compr. Cancer Netw. 2020, 18, 591-598. [CrossRef] [PubMed] 